\title{
TEST-RETEST RELIABILITY OF SELECTED HBSC ITEMS MEASURING PROBLEM BEHAVIOUR AMONG SLOVAK AND CZECH ADOLESCENTS
}

\author{
Jana Holubčíková1, Michal Kudláček ${ }^{2,3}$, Jan Širůček ${ }^{4}$, Andrea Madarasová Gecková1, 5 \\ ${ }^{1}$ Department of Health Psychology, Faculty of Medicine, P. J. Šafárik University in Košice, Košice, Slovak Republic \\ ${ }^{2}$ Department of Recreation and Leisure Studies, Faculty of Physical Culture, Palacký University Olomouc, Olomouc, Czech Republic \\ ${ }^{3}$ Centre for Kinanthropology Research, Institute of Active Lifestyle, Faculty of Physical Culture, Palacký University Olomouc, Olomouc, Czech \\ Republic \\ ${ }^{4}$ Department of Psychology, Faculty of Social Studies, Masaryk University, Brno, Czech Republic \\ ${ }^{5}$ Olomouc University Social Health Institute, Palacký University Olomouc, Olomouc, Czech Republic
}

\section{SUMMARY}

Objectives: The purpose of this study was to assess the test-retest reliability of selected items based on self-reports, measuring problem behaviour in the Slovak and Czech version of the HBSC survey questionnaire.

Methods: The data from test-retest study, based on an international Health Behaviour in School-aged Children (HBSC) study and consistent with its methodology, were analysed.

A sample of 580 primary school pupils ( $51.2 \%$ of boys), grades five and nine participated in a test and retest with a four-week interval. Six items concerning problem behaviour were evaluated overall and stratified by gender and age.

Results: Analyses of test-retest reliability indicated modest ( 0.30 to 0.49$)$, moderate $(0.50$ to 0.69$)$, or high ( 0.70 to 1.00$)$ reliability across nearly all questions, with some reliability differences in analyses by gender and age. In general, findings of present study suggest the moderate reliability of measures of smoking, drunkenness, fighting and negative relationship to school, modest reliability of measures of bullying behaviour, and low reliability of measure of truancy.

Conclusions: The overall findings of this study suggest that most of selected indicators in the HBSC survey questionnaire have satisfactory testretest reliability. Further test-retest studies in a large and diverse sample, as well as validity studies, should be considered for the future HBSC study.

Key words: test-retest reliability, problem behaviour, adolescence

Address for correspondence: J. Holubčíková, Department of Health Psychology, Faculty of Medicine, P. J. Šafárik University in Košice, Trieda SNP 1, 04011 Košice, Slovak Republic. E-mail: jana.holubcikova@upjs.sk

https://doi.org/10.21101/cejph.a4662

\section{INTRODUCTION}

Adolescent problem behaviour such as substance use, aggressive behaviour or problems in school is an increasing concern in many countries. Previous evidence has shown that different risk or problem behaviours in adolescence are interrelated (1-6). During this period, adolescents start to experiment with alcohol, smoking and other problem behaviours and among some of them these behaviours pertain to adulthood, which is associated with negative health consequences $(7,8)$. As early adolescence is characteristic by experiments, the nature and prevalence of different types of problem behaviours is difficult to measure. Early identification of problem behaviour during this period is important for creating appropriate public health interventions and to decrease the prevalence of these behaviours. Therefore, adequate measurement of different types of problem behaviours is necessary to determine the prevalence and factors related to the occurrence and course of problem behaviours during this period of life.

Several European studies (European School Survey Project on Alcohol and Other Drugs, International Self-Report Delin- quency Study, Health Behaviour in School-aged Children, etc.) are focused on monitoring problem behaviour of adolescents using self-reports of adolescents. Establishing the reliability of self-reported measures is a necessary step in determining the utility of methodology of particular study and in studying behaviours in the context of risk and protective factors. The Health Behaviour in School-aged Children (HBSC) study is among the first large-scale international surveys on adolescent health. The present paper uses results from a sample of Czech and Slovak elementary school students to test problem behaviour. The aim of the present study was to assess the reliability of self-reports measuring these behaviours in HBSC questionnaire.

\section{MATERIALS AND METHODS}

\section{Sample and Procedure}

This test-retest study is based on an international Health Behaviour in School-aged Children (HBSC) study and it is consist- 
ent with its methodology. The testing and re-testing procedures were conducted in November and December 2013 in the Czech Republic and Slovakia. This study was preceded by a pilot study which included the administration of questionnaires and focus groups in both countries. Based on data obtained in the pilot study the final set of questions was compiled. We contacted 12 larger and smaller primary schools located in rural as well as in urban areas in Olomouc region, Czech Republic (7 schools), and Kosice region, Slovakia ( 5 schools). These were randomly chosen to create a representative sample. All of the contacted schools agreed to participate. Questionnaires were administrated in 5th and 9th grades by trained research assistants in the absence of a teacher during regular class time.

In the first part of data collection (test), we obtained data from 406 adolescents in the Czech Republic (response rate 83.20\%) and 258 adolescents in Slovakia (response rate 74.14\%). Non-response was primarily due to illness and parental disapproval for the participation of their children. Second part of data collection (retest) was conducted 4 weeks after the first one. We obtained data from 353 adolescents in the Czech Republic (53 dropped out) and 227 adolescents in Slovakia (31 dropped out) who participated also in the first part of data collection (test). The final sample consisted of 353 Czech (51.9\% of boys) and 227 Slovak (52.9\% of boys) primary school pupils, grades five and nine.

The study was conducted in compliance with the ethical requirements formulated by the Agreement on Human Rights and Biomedicine* and the Declaration of Helsinki principles as well as with legal and regulatory requirements which apply to both countries. The study was approved by the relevant ethics committees of the participating universities, i.e. P. J. Šafárik University in Košice, Slovak Republic, and Palacký University Olomouc, Czech Republic. Parents were informed about the study via the school administration and could opt out if they disagreed with it. Participation in the study was fully voluntary and anonymous with no explicit incentives provided for participation. The study in the Czech Republic was approved by the Ministry of Health and the National Institute of Public Health. Czech legislation did not require this study to be approved by an Ethics Committee as students completed the questionnaire anonymously.

\section{Measures}

We used data related to adolescents' reports of several types of problem behaviour. Table 1 provides an overview of the measures and the dichotomisation of response alternatives used in this study regarding smoking status, drunkenness, fighting, bullying, and truancy.

Smoking status was defined on the basis of the question "How often do you smoke tobacco at present?" Possible responses included 'every day'; 'at least once a week, but not every day'; less than once a week'; or 'never'.

Drunkenness has been assessed with question: "Have you ever had so much alcohol that you were really drunk?" The following multiple-choice answers were offered: no, never; yes, once; yes, 2-3 times; yes, 4-10 times; yes, more than 10 times.

Fighting was defined according to the answer to the question "In the last 12 months, how many times were you involved in physical fighting?"

Bullying behaviour of respondents was measured by the question from revised Olweus Bully/Victim Questionnaire (9). After having read a standard definition of bullying, respondents were asked about their involvement in bullying - how often they had bullied others in the last few months. Responses were rated on a five-point scale ("I haven't been bullied/bullied other students at school in the past couple of months", "only once or twice", "two or three times a month", "about once a week", "several times a week").

Truancy was measured by asking: "How many days have you skipped classes or school (without permission) this term?" Response options to this question $(0,1,2,3$ days or 4 days or more) were recorded in three categories; not at all; 1-3 days; and 4 or more days.

School liking was measured by the question: "How do you feel about school at present?" With possible responses: "I like it a lot"; "I like it a bit"; "I don't like it very much"; and "I don't like it at all".

Table 1. Selected items, response alternatives and dichotomisation of HBSC survey questionnaire used in test-retest study

\begin{tabular}{|c|c|c|c|}
\hline & Items & Response alternatives & Dichotomisation of reference group \\
\hline Smoking & $\begin{array}{l}\text { "How often do you smoke tobacco at } \\
\text { present?" }\end{array}$ & $\begin{array}{l}\text { "every day"; "at least once a week, but not } \\
\text { every day"; "less than once a week"; or "never" }\end{array}$ & at least once and more \\
\hline Drunkenness & $\begin{array}{l}\text { "Have you ever had so much alcohol that } \\
\text { you were really drunk?" }\end{array}$ & $\begin{array}{l}\text { "no, never"; "yes, once"; "yes, } 2-3 \text { times"; "yes, } \\
\text { 4-10 times"; "yes, more than } 10 \text { times" }\end{array}$ & once and more \\
\hline Fighting & $\begin{array}{l}\text { "In the last } 12 \text { months, how many times } \\
\text { were you involved in physical fighting?" }\end{array}$ & $\begin{array}{l}\text { "I have not been in a physical fight in the past } \\
12 \text { month"; " } 1 \text { time"; " } 2 \text { times"; } \\
\text { "3 times"; "4 times or more" }\end{array}$ & 1 time and more \\
\hline Bullying & $\begin{array}{l}\text { After having read a standard definition of } \\
\text { bullying, respondents were asked: "How of- } \\
\text { ten have you taken part in bullying another } \\
\text { student(s) at school in the past couple of } \\
\text { months?" }\end{array}$ & $\begin{array}{l}\text { "I have not been bullied/bullied other students } \\
\text { at school in the past couple of months"; "only } \\
\text { once or twice"; "two or three times a month"; } \\
\text { "about once a week"; "several times a week" }\end{array}$ & two or three times a months and more \\
\hline Truancy & $\begin{array}{l}\text { "How many days have you skipped classes } \\
\text { or school (without permission) this term?" }\end{array}$ & " $0,1,2,3$ days or 4 days or more" & one day and more \\
\hline School liking & "How do you feel about school at present?" & $\begin{array}{l}\text { "I like it a lot"; "I like it a bit"; "don't like it very } \\
\text { much"; and "don't like it at all" }\end{array}$ & I don't like it very much or not at all \\
\hline
\end{tabular}

$*$ 40/2000 Coll. 


\section{Statistical Analyses}

Firstly, we described socio-demographic characteristics: gender, grade and country (Table 2). Then, the prevalence of all variables describing problem behaviour were analysed: drunkenness, smoking, fighting, bullying, truancy, and school dislike overall (Table 3), and stratified by gender and grade category (Table 4).

To indicate test-retest reliability of variables, we used kappa statistics (k) which measure agreement, thus, it is preferable for assessing reliability than measures such as Pearson Product-

Table 2. Description of the sample: gender, grade and country $(N=580)$

\begin{tabular}{|c|c|c|c|c|c|}
\hline \multicolumn{2}{|c|}{$\begin{array}{c}\text { Gender } \\
\mathrm{n}(\%)\end{array}$} & \multicolumn{2}{c|}{$\begin{array}{c}\text { Grade } \\
\mathrm{n}(\%)\end{array}$} & \multicolumn{2}{c|}{$\begin{array}{c}\text { Country } \\
\mathrm{n}(\%)\end{array}$} \\
\hline Boys & Girls & 5 th & 9 th & Slovak Republic & Czech Republic \\
\hline $297(51.2)$ & $283(48.8)$ & $309(53.3)$ & $271(46.7)$ & $227(39.1)$ & $353(60.9)$ \\
\hline
\end{tabular}

Tables 3. Prevalence of problem behaviour and test-retest reliability of measures of problem behaviour among adolescents (kappa measure agreement; kappa, SE) $(N=580)$

\begin{tabular}{|l|c|c|c|c|}
\hline & $\begin{array}{c}\text { Test } \\
\mathbf{n}(\%)\end{array}$ & $\begin{array}{c}\text { Retest } \\
\mathbf{n}(\%)\end{array}$ & kappa & SE \\
\hline Experience with smoking & $58(10.1)$ & $42(7.5)$ & $0.62^{* \star *}$ & 0.064 \\
\hline Being drunk & $125(21.7)$ & $144(24.8)$ & $0.68^{* *}$ & 0.036 \\
\hline Fighting in last year & $186(32.5)$ & $176(30.4)$ & $0.60^{* \star *}$ & 0.036 \\
\hline Bullying others & $29(6.0)$ & $25(5.0)$ & $0.39^{* \star *}$ & 0.100 \\
\hline Truancy & $66(11.5)$ & $61(10.6)$ & $0.27^{* \star *}$ & 0.059 \\
\hline Negative relationship to school & $177(30.8)$ & $172(29.8)$ & $0.61^{* * *}$ & 0.036 \\
\hline
\end{tabular}

Only valid percentages are presented; missing values:

Test: gender $n=0(0 \%)$, country $=0(0 \%)$, class $n=0(0 \%)$, physical fight $n=7(1.2 \%)$, bullying $n=16(2.8 \%)$, smoking $n=3(0.5 \%)$, drunkenness $n=4(0.7 \%)$, truancy $n=6$ $(1.0 \%)$, relationship to school $n=6(1.0 \%)$

Retest: gender $n=0(0 \%)$, country $=0(0 \%)$, class $n=0(0 \%)$, physical fight $n=1(0.2 \%)$, bullying $n=7(1.2 \%)$, smoking $n=1(0.2 \%)$, drunkenness $n=0(0 \%)$, truancy $n=3$ $(0.5 \%)$, relationship to school $n=2(0.3 \%)$

${ }^{* * *} \mathrm{p}<0.001$

Table 4. Prevalence of problem behaviour and test-retest reliability of measures of problem behaviour among adolescents (kappa measure agreement; kappa, SE) stratified by gender and grade $(N=580)$

\begin{tabular}{|c|c|c|c|c|c|c|c|c|}
\hline \multirow[b]{2}{*}{ Gender } & \multicolumn{4}{|c|}{ Boys $(n=297)$} & \multicolumn{4}{|c|}{ Girls $(n=283)$} \\
\hline & $\begin{array}{c}\text { Test } \\
\mathbf{n}(\%)\end{array}$ & $\begin{array}{c}\text { Retest } \\
\mathrm{n}(\%)\end{array}$ & kappa & SE & $\begin{array}{c}\text { Test } \\
\mathrm{n}(\%)\end{array}$ & $\begin{array}{c}\text { Retest } \\
\mathrm{n}(\%)\end{array}$ & kappa & SE \\
\hline Experience with smoking & $28(9.5)$ & $19(6.6)$ & $0.624^{* \star *}$ & 0.09 & $30(10.6)$ & $23(8.4)$ & $0.621^{* * *}$ & 0.09 \\
\hline Being drunk & $70(23.7)$ & $77(25.9)$ & $0.654^{* * *}$ & 0.05 & $55(19.6)$ & $67(23.7)$ & $0.716^{\star * *}$ & 0.05 \\
\hline Fighting in last year & $130(44.4)$ & $127(42.8)$ & $0.611^{* * *}$ & 0.04 & $56(20)$ & $49(17.4)$ & $0.497^{* * *}$ & 0.06 \\
\hline Bullying others & $19(8.2)$ & $20(8.3)$ & $0.558^{* \star *}$ & 0.12 & $10(4.0)$ & $5(2.0)$ & $0.121^{*}$ & 0.12 \\
\hline Truancy & $39(13.4)$ & $38(12.9)$ & $0.280^{* * *}$ & 0.07 & $27(9.6)$ & $23(8.2)$ & $0.254^{* * *}$ & 0.09 \\
\hline Negative relationship to school & $104(35.4)$ & $103(34.8)$ & $0.647^{* \star *}$ & 0.04 & $73(26.1)$ & $69(24.5)$ & $0.555^{* * *}$ & 0.05 \\
\hline \multirow[b]{2}{*}{ Grade } & \multicolumn{4}{|c|}{5 th grade $(n=309)$} & \multicolumn{4}{|c|}{ 9th grade $(n=271)$} \\
\hline & $\begin{array}{c}\text { Test } \\
\mathrm{n}(\%)\end{array}$ & $\begin{array}{c}\text { Retest } \\
\mathrm{n}(\%)\end{array}$ & kappa & SE & $\begin{array}{c}\text { Test } \\
\mathrm{n}(\%)\end{array}$ & $\begin{array}{c}\text { Retest } \\
\mathrm{n}(\%)\end{array}$ & kappa & SE \\
\hline Experience with smoking & $9(2.9)$ & $9(2.9)$ & $0.428^{* * *}$ & 0.14 & 49 (18.1) & $33(13.1)$ & $0.664^{* * *}$ & 0.07 \\
\hline Being drunk & $25(8.2)$ & $27(8.7)$ & $0.251^{* * *}$ & 0.08 & $100(37.0)$ & $117(43.2)$ & $0.769^{\star \star *}$ & 0.03 \\
\hline Fighting in last year & $94(30.9)$ & $100(32.5)$ & $0.647^{* * *}$ & 0.04 & $92(34.2)$ & $76(28.0)$ & $0.549^{* * *}$ & 0.05 \\
\hline Bullying others & $14(5.4)$ & $10(3.9)$ & $0.263^{* * *}$ & 0.16 & $15(6.8)$ & $15(6.3)$ & $0.469^{* * *}$ & 0.13 \\
\hline Truancy & $36(11.8)$ & $34(11.1)$ & $0.395^{* \star *}$ & 0.08 & 30 (11.2) & $27(10.0)$ & $0.124^{*}$ & 0.07 \\
\hline Negative relationship to school & $61(20.1)$ & $55(17.9)$ & $0.699^{\star * *}$ & 0.05 & $116(43.0)$ & $117(43.2)$ & $0.547^{\star \star *}$ & 0.05 \\
\hline
\end{tabular}

Only valid percentages are presented; missing values:

Test: gender $n=0(0 \%)$, class $n=0(0 \%)$, physical fight $n=7(1.2 \%)$, bullying $n=16(2.8 \%)$, smoking $n=3(0.5 \%)$, drunkenness $n=4(0.7 \%)$, truancy $n=6(1.0 \%)$, relationship to school $n=6(1.0 \%)$

Retest: gender $n=0(0 \%)$, class $n=0(0 \%)$, physical fight $n=1(0.2 \%)$, bullying $n=7(1.2 \%)$, smoking $n=1(0.2 \%)$, drunkenness $n=0(0 \%)$, truancy $n=3(0.5 \%)$, relationship

to school $n=2(0.3 \%)$

${ }^{*} p<0.05 ;{ }^{* * *} p<0.001$ 
Moment correlations, which measure association $(10,11)$. Reliability was rated as modest (0.30-0.49), moderate (0.50-0.69), or high (0.70-1.00). We compared responses of the respondents from the first trial (test) with the second trial (retest). The kappa coefficients of all variables are shown in Table 3 and 4 . To ensure comparability of the reliability of each item between gender and grade groups, all analyses were stratified.

\section{RESULTS}

In general, the prevalence of problem behaviour does not differ significantly between test and retest. When comparing gender, boys reported significantly higher level of fighting, bullying and negative relationship to school. The differences between grade groups emerged mainly in smoking status, drunkenness and negative relationship to school.

Overall, the results of the kappa statistics showed moderate reliability of measures of smoking, drunkenness, fighting, and negative relationship to school. Modest reliability was found for measures of bullying behaviour. The kappa coefficient of measure of truancy showed as unreliable. After stratification, changes in the level of reliability of some measures emerged. Taking in account gender differences, the measure of fighting became less reliable among girls and the measure of bullying behaviour became more reliable among boys. After stratification according to grade, the reliability of measures of smoking and drunkenness were found to be modest among younger adolescents, while this reliability remained moderate among older ones. The reliability of bullying measure was found to be better among adolescents from 9th grade.

\section{DISCUSSION}

Overall, the test-retest reliability results showed moderate to almost perfect agreement for most of the items, except for one item related to truancy. When considering gender and grade differences, it seems that the reliability of some items is changing with respect to these characteristic. The findings suggest that all these indicators except one (truancy) are reliable to measure problem behaviour of school-aged children in Slovakia and the Czech Republic.

A few gender and age group differences were observed in the reliability of some indicators measuring problem behaviour among respondents. In general, the measure of smoking status showed a moderate level of reliability especially among older adolescents, in accordance with the previous evidence that selfreported smoking prevalence has been considered as a good indicator of the actual smoking status compared with biochemical validated smoking prevalence (12). In addition, test-retest reliability of measures of smoking status and drunkenness was explored in the study from China (13), and they found moderate reliability among 15-year olds.

Self-reported alcohol use is considered to be highly reliable and accurate (14) which is in line with our results. The measure of drunkenness showed moderate level of reliability, and after stratification age differences emerged. The reliability of the measure remained moderate among 9th grade adolescents, whereas it became modest among 5 th grade adolescents.
The measure of frequency of fighting used in HBSC survey has been well validated and reliability ascertained with extensive use in the US Youth Risk Behaviour Survey $(15,16)$. We also found the moderate reliability for this item.

The role of adolescents in bullying was measured by the revised Olweus Bully/Victim Questionnaire (9). This measure is frequently used in the HBSC survey and in a variety of reports and peer-review publications at national and cross-national levels (17-19). Recent literature review identified this measure to have the strongest support for its psychometric soundness (20) but our results suggest modest reliability. After stratification of gender and grade category, we found that reliability increased to moderate in boys and in older adolescents.

Truancy was measured by the question which was created and used in the International self-report delinquency study. This measure was added to Slovak HBSC study in 1993. Our results suggest very low reliability of this measure. After stratification on grade, the reliability improved to the modest level among 5th grade adolescents and decreased for 9th grade.

Measure of the relationship to school, which has been included in the HBSC survey since 1985/86, has been found to be a powerful correlate of health behaviours and health perceptions $(21,22)$. We found moderate reliability of this measure with no significant differences between gender and grade categories.

\section{Strengths and Limitations}

The major strength of this study is the use of methodology of the international Health Behaviour in School-aged Children (HBSC) survey. In addition, this is the first study dealing with test-retest reliability of selected HBSC measures of problem behaviour in central Europe, particularly among Slovak and Czech of adolescents. Limitation of this study is that it was conducted on a convenience sample so its representativeness and generalizability relative to the population were undetermined.

\section{Implications}

Accurate measurement techniques are essential to the ability to identify problem behaviour among adolescents and to create effective prevention and intervention strategies. Further test-retest studies in a large and diverse sample, as well as validity studies, should be considered for the future HBSC study.

\section{CONCLUSION}

To conclude, present study explored the test-retest reliability of selected items used in the international HBSC study measuring problem behaviour of adolescents. The measures of smoking status, frequency of drunkenness, fighting, and negative relationship to school showed moderate level of test-retest reliability. Measure of bullying behaviour indicated satisfactory reliability among boys and measure of truancy was found to have poor reliability.

\section{Acknowledgements}

This work was partially supported by the Research and Development Support Agency under Contract No. APVV 0032-11, APVV-15-0012 and was also partially funded within the framework of the project "Social 
determinants of health in socially and physically disadvantaged and other groups of population" of The Ministry of Education, Youth and Sports of the Czech Republic CZ.1.07/2.3.00/20.0063. This work was also supported by the Faculty of Physical Culture, Palacký University in Olomouc within the project "Children's leisure time - HBSC study" FTK_2013_020.

\section{Conflict of Interests}

None declared

\section{REFERENCES}

1. Diehl K, Thiel A, Zipfel S, Mayer J, Litaker DG, Schneider S. How healthy is the behavior of young athletes? A systematic literature review and meta-analyses. J Sports Sci Med. 2012;11(2):201-20.

2. Kaai SC, Leatherdale ST, Manske SR, Brown KS. Using student and school factors to differentiate adolescent current smokers from experimental smokers in Canada: a multilevel analysis. Prev Med. 2013;57(2):113-9.

3. Kwan B, Frankish J, Rootman I, et al. The development and validation of measures of "health literacy" in different populations. Vancouver: University of British Columbia; 2006.

4. Lisha NE, Sussman S. Relationship of high school and college sports participation with alcohol, tobacco, and illicit drug use: a review. Addict Behav. 2010;35(5):399-407.

5. Townshend TG. Youth, alcohol and place-based leisure behaviours: a study of two locations in England. Soc Sci Med. 2013;91:153-61.

6. White HR, Fite P, Pardini D, Mun EY, Loeber R. Moderators of the dynamic link between alcohol use and aggressive behavior among adolescent males. J Abnorm Child Psychol. 2013;41(2):211-22.

7. Cook EC, Pflieger JC, Connell AM, Connell CM. Do specific transitional patterns of antisocial behavior during adolescence increase risk for problems in young adulthood? J Abnorm Child Psychol. 2015;43(1):95-106.

8. Dubow EF, Boxer P, Huesmann LR. Childhood and adolescent predictors of early and middle adulthood alcohol use and problem drinking: the Columbia County Longitudinal Study. Addiction. 2008;103(Suppl 1):36-47.

9. Solberg ME, Olweus D. Prevalence estimation of school bullying with the Olweus Bully/Victim Questionnaire. Aggress Behav. 2003;29(3):239-68.

10. Shrout PE, Fleiss JL. Intraclass correlations: uses in assessing rater reliability. Psychol Bull. 1979;86(2):420-8.

11. Tan SB, Wee SB, Cheung YB. Agreement or prediction: asking and answering the right question. Ann Acad Med Singapore. 2002;31(3):405-7.
12. Currie C, Zanotti C, Morgan A, Currie D, de Looze M, Roberts C, et al, editors. Social determinants of health and well-being among young people: Health Behaviour in School-aged Children (HBSC) study: international report from the 2009/2010 survey. Health policy for children and adolescents, no 6. Copenhagen: WHO Regional Office for Europe; 2012.

13. Liu Y, Wang M, Tynjälä J, Lv Y, Villberg J, Zhang Z, et al. Test-retest reliability of selected items of Health Behaviour in School-aged Children (HBSC) survey questionnaire in Beijing, China. BMC Med Res Methodol. 2010 Aug 10;10:73. doi: 10.1186/1471-2288-10-73.

14. Campanelli PC, Dielman TE, Shope JT. Validity of adolescents' selfreports of alcohol use and misuse using a bogus pipeline procedure. Adolescence. 1987;22(85):7-22.

15. Kann L, Kinchen SA, Williams BI, Ross JG, Lowry R, Grundbaum JA, et al. Youth risk behavior surveillance - United States, 1999. MMWR CDC Surveill Summ. 2000 Jun 9;49(5):1-32.

16. Brener ND, Collins JL, Kann L, Warren CW, Williams BI. Reliability of the Youth Risk Behavior Survey questionnaire. Am J Epidemiol. 1995;141(6):575-80.

17. Brixval CS, Rayce SLB, Rasmussen M, Holstein BE, Due P. Overweight, body image and bullying - an epidemiological study of 11- to 15-years olds. Eur J Public Health. 2012;22(1):126-30.

18. Craig W, Harel-Fisch Y, Fogel-Grinvald H, Dostaler S, Hetland J, SimonsMorton B, et al. A cross-national profile of bullying and victimization among adolescents in 40 countries. Int J Public Health. 2009;54 Suppl 2:216-24.

19. Harel-Fisch Y, Walsh SD, Fogel-Grinvald H, Amitai G, Pickett W, Molcho $\mathrm{M}$, et al. Negative school perceptions and involvement in school bullying: a universal relationship across 40 countries. J Adolesc. 2011;34(4):639-52.

20. Vessey J, Strout TD, DiFazio RL, Walker A. Measuring the youth bullying experience: a systematic review of the psychometric properties of available instruments. J Sch Health. 2014;84(12):819-43.

21. Samdal O, Wold B, Torsheim T. Rationale for school items: the relationship between students' perception of school and their reported health and quality of life. In: Currie C, editor. Health Behaviour in School-aged Children. Research protocol for the 1997-98 survey. Edinburgh: University of Edinburgh; 1998. p. 51-9.

22. Wold B, Aaro L, Smith C. Health Behaviour in School-aged Children: a WHO cross-national study. Research protocol for the 1993/94 survey. Bergen: University of Bergen; 1994.

Received December 14, 2015 Accepted in revised form August 6, 2018 